\title{
Universal Current-Mode Biquad Employing Dual Output Current Conveyors and MO-CCCA with Grounded Passive Elements
}

\author{
Kasim Karam Abdalla \\ Department of Electrical Engineering, Engineering Colloge, University of Babylon, Hilla, Iraq \\ Email: kasimkaa.11@gmail.com
}

Received May 17, 2012; revised November 6, 2012; accepted November 13, 2012

\begin{abstract}
A new universal multiple input multiple output (MIMO) type current-mode biquad employing two dual output current conveyors (DOCCII), one multiple output current controlled current amplifier (MOCCCA) and four passive grounded elements is proposed which can realize all the five basic filtering functions namely, low-pass (LP), high-pass (HP), band-pass (BP), band-stop (BR) and all-pass (AP) in current mode from the same configuration. The centre frequency $\omega_{o}$ can be set by the passive elements of the circuit and the quality factor $Q_{o}$ is electronically tunable through bias currents of the MOCCCA. Therefore, the biquad filter has independent tenability for the $\omega_{o}$ and $Q_{o}$. The active and passive sensitivities of $Q_{o}$ and $\omega_{o}$ are low. The workability of the new configuration has been demonstrated by PSPICE simulation results based upon a CMOS CCII in $0.35 \mu \mathrm{m}$ technology.
\end{abstract}

Keywords: Current-Mode Filters; Current Conveyors; Analog Circuit Design; CMOS Circuits

\section{Introduction}

Recently, Chunhua, Hiaguang and Yan presented two new universal multiple input single output (MISO) current-mode $(\mathrm{CM})$ biquadatic filters using one MOCCCA, two grounded capacitors (GC) and two grounded resistors (GR) and realize all the five generic filter responses in $\mathrm{CM}$ (i.e. with current as input and current as output) [1].

The purpose of this paper is to introduce a new configuration which although uses exactly same number of active and passive components but in contrast to the circuit of reference [1] realizes a MIMO-type biquad and hence, does not require any additional hardware to duplicate/invert current inputs which is required in case of MISO-type filters of [1].

In the literature there are SIMO-type filter circuits which have three active devices but suffer from the independent tunability as in [2-5] or have more passive or active elements as in [4-9]. The circuits in [10-12] need double inputs and outputs to realize all five generic filters. The circuit in [13] has two MO-CCCIIs and one DOCCCII, the draw back of this circuit is the control currents $I_{o i}, i=1,2,3$ are temperature dependent. The circuit in [14] has two MO-CCCIIs and one MOCCCA but realizes only SIMO-type biquad.

\section{The Proposed Configuration}

The proposed configuration is shown in Figure 1.

Assuming the CCIIs to be characterized by

$$
\left[\begin{array}{c}
I_{Y} \\
V_{X} \\
I_{Z}
\end{array}\right]=\left[\begin{array}{ccc}
0 & 0 & 0 \\
1 & 0 & 0 \\
0 & \pm 1 & 0
\end{array}\right]\left[\begin{array}{c}
V_{Y} \\
I_{X} \\
V_{Z}
\end{array}\right]
$$

The symbolic notation of MO-CCCA is given in Figure 2(a), where $i$ represent input, $\left(I_{o 1}-I_{o n}\right)$ are $\mathrm{n}$ outputs respectively, and $I_{A}$ and $I_{B}$ denote DC bias currents. Figure 2(b) is a CMOS realization of MO-CCCA. Here $I_{i}$ denotes the input signal; $I_{o 1}, I_{o 2}, I_{03}$ are the three output currents, respectively.

If the channel lengths of $M_{5}-M_{8}$ are all $\mathrm{n}$ times of that of $M_{4}$, and the channel size of $M_{17}$ is n times that of $M_{18}$, namely

$$
\begin{aligned}
& (W / L)_{M_{5}} /(W / L)_{M_{4}} \\
& =(W / L)_{M_{6}} /(W / L)_{M_{4}}=(W / L)_{M_{7}} /(W / L)_{M_{4}} \\
& =(W / L)_{M_{8}} /(W / L)_{M_{4}}=(W / L)_{M_{17}} /(W / L)_{M_{18}}=n,
\end{aligned}
$$

the output current expressions can be obtained as

$$
I_{o 1}=I_{o 2}=\cdots=I_{o n}=\frac{n I_{B}}{2 I_{A}}=K I_{i}
$$




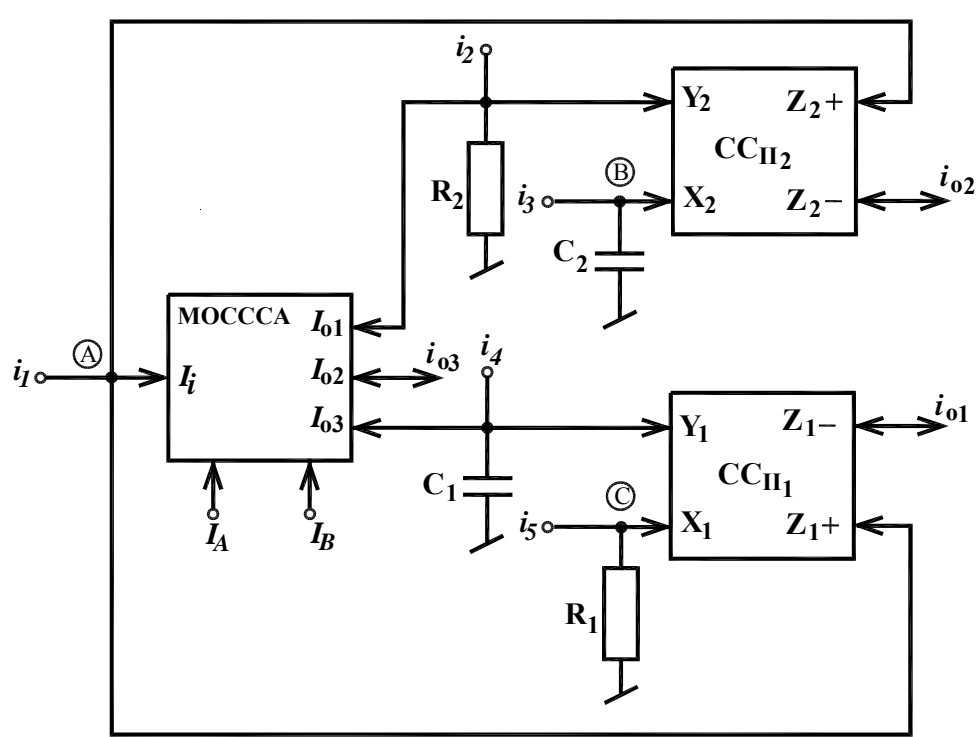

Figure 1. The proposed configuration.

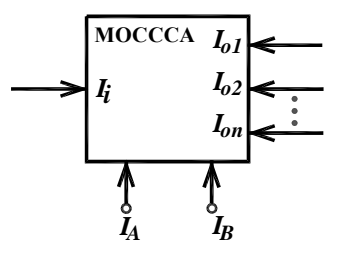

(a)

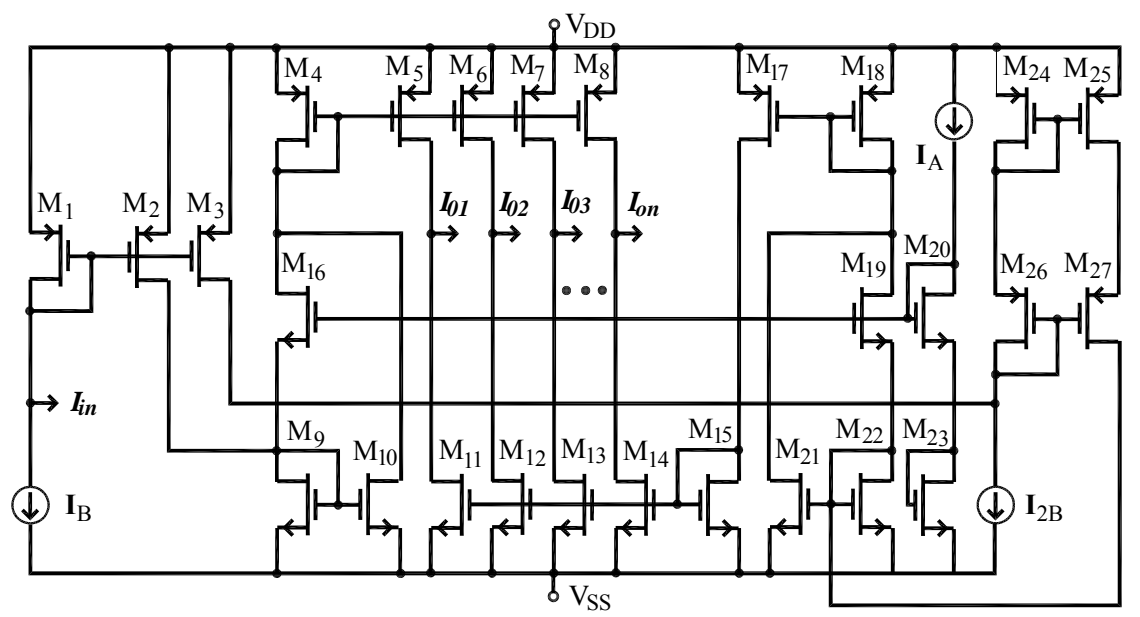

(b)

Figure 2. (a) Symbolic notation of MO-CCCA (b) CMOS realization of the MO-CCCA.

where $K$ represents the current gain. It is clear from Equation (2) that the value of $K$ can be set by $I_{B}$ and $I_{A}$. Consider now the following special cases:

\subsection{MISO Type:}

When $i_{1}, i_{2}, i_{3}, i_{4}$ and $i_{5}$ are input currents and taking $i_{03}$ as output current, then a routine analysis of the circuit reveals the following expression of the output current $i_{03}$ in terms of the five input currents $i_{1}, i_{2}, i_{3}, i_{4}$ and $i_{5}$ :

$$
i_{03}=\frac{1}{\Delta}\left[i_{2} s^{2} C_{1} C_{2}+i_{4} G_{1} G_{2}+\left(i_{1}-i_{3}-i_{5}\right) s C_{1} G_{2}\right]
$$

where $\Delta=s^{2} C_{1} C_{2}+\frac{1}{K} s C_{1} G_{2}+G_{1} G_{2}, G_{1}=1 / R_{1}$ and $G_{2}=1 / R_{2}$.

Then, the various filter responses can be realized from 
the circuit are:

LPF: when $i_{4}=i_{\text {in }}$ (non-inv.) and

$i_{1}=i_{2}=i_{3}=i_{5}=0$.

HPF: when $i_{2}=i_{\text {in }}$ and $i_{1}=i_{3}=i_{4}=i_{5}=0$.

BPF: when $i_{1}=i_{\text {in }}$ and $i_{2}=i_{3}=i_{4}=i_{5}=0$ or $i_{3}=i_{\text {in }}$ and $i_{1}=i_{2}=i_{4}=i_{5}=0$ or $i_{5}=i_{\text {in }}$ and

$i_{1}=i_{2}=i_{3}=i_{4}=0$.

Notch: when $i_{2}=i_{4}=i_{\text {in }}$ and $i_{1}=i_{3}=i_{5}=0$.

APF: when $i_{2}=i_{3}=i_{4}=i_{\text {in }}$ and $i_{1}=i_{5}=0$ or $i_{2}=i_{4}=i_{5}=i_{\text {in }}$ and $i_{1}=i_{3}=0$.

\subsection{SIMO Type}

If $i_{1}$ is input current, $i_{2}=i_{3}=i_{4}=i_{5}=0$ (open circuited) then, the various filter responses realized are given by:

$$
\begin{gathered}
\text { LPF: } \frac{i_{01}}{i_{1}}=\frac{-1}{\Delta}\left[G_{1} G_{2}\right] \\
\text { HPF: } \frac{i_{02}}{i_{1}}=\frac{-1}{\Delta}\left[s^{2} C_{1} C_{2}\right] \\
\text { BPF: } \frac{i_{03}}{i_{1}}=\frac{1}{\Delta}\left[s C_{1} G_{2}\right]
\end{gathered}
$$

$$
\text { Notch: } \frac{i_{01}+i_{02}}{i_{1}}=\frac{-1}{\Delta}\left[s^{2} C_{1} C_{2}+G_{1} G_{2}\right]
$$

$\mathrm{APF}: \frac{i_{01}+i_{02}+i_{03}}{i_{1}}=\frac{-1}{\Delta}\left[s^{2} C_{1} C_{2}-s C_{1} G_{1}+G_{1} G_{2}\right]$

The various parameters of the realized filters are given by

$$
\omega_{o}=\sqrt{\frac{1}{C_{1} C_{2} R_{1} R_{2}}}, B W=\frac{1}{K C_{2} R_{2}}, Q_{o}=K \sqrt{\frac{C_{2} R_{2}}{C_{1} R_{1}}}
$$

From Equation (9), the centre frequency $\omega_{o}$ can be set by varying $R_{1}$ without disturbing $\omega_{o} / Q_{o}$. The $Q_{o}$ can also be set by $I_{B}$ and $I_{A}$ without disturbing $\omega_{o}$. Therefore, the biquad filter has independent tenability for the $\omega_{0}$ and $Q_{o}$.

From the above, the active and passive sensitivities of the transfer function are given as

$$
\begin{aligned}
& S_{C_{1}}^{\omega_{o}}=S_{C_{2}}^{\omega_{o}}=S_{R_{1}}^{\omega_{o}}=S_{R_{2}}^{\omega_{o}}=-\frac{1}{2}, S_{C_{2}}^{Q_{o}}=S_{R_{2}}^{Q_{o}}=\frac{1}{2} \\
& S_{C_{1}}^{Q_{o}}=S_{R_{1}}^{Q_{o}}=-\frac{1}{2}, S_{K}^{Q_{o}}=1
\end{aligned}
$$

The active and passive sensitivities of $\omega_{o}$ and $Q_{o}$ are found to be in the range $-\frac{1}{2} \leq S_{x}^{F} \leq 1$, and the circuit, thus, enjoys low sensitivities.

\section{Simulation Results}

To verify the validity of the various modes of operation of the proposed configuration, circuit simulation of the current mode filters (MISO and SIMO) have been carried out using the CMOS CCII implementation with multiple outputs shown in Figure 3 (as in [15], modified from [16]).

The model parameters of $n$-channel and p-channel MOSFETs are given in [17], whereas aspect ratios of the CCII MOSFETs are shown in Table 1, and aspect ratios of the MO-CCCA MOSFETs are shown in Table 2.

The CMOS CCII was biased with DC power supply voltages $V_{D D}=+1.5 \mathrm{~V}, V_{S S}=-1.5 \mathrm{~V}, V_{1}=-0.5 \mathrm{~V}$, and $V_{2}=-0.9 \mathrm{~V}$.

To achieve the MISO type filters with $f_{o}=1 \mathrm{MHz}$ and quality factor of $Q_{o}=1$, the component values were selected $K=1\left(n=1, I_{A}=50 \mu \mathrm{A}, I_{B}=100 \mu \mathrm{A}\right)$, $R_{1}=R_{2}=1 \mathrm{k} \Omega$ and $C_{1}=C_{2}=159 \mathrm{pF}$. The frequency responses of LPF, BPF, HPF, Notch and APF (theoretical and simulation) are shown in Figure 4.

To test the input dynamic range of the proposed filters, the simulation of the band-pass filter as an example has

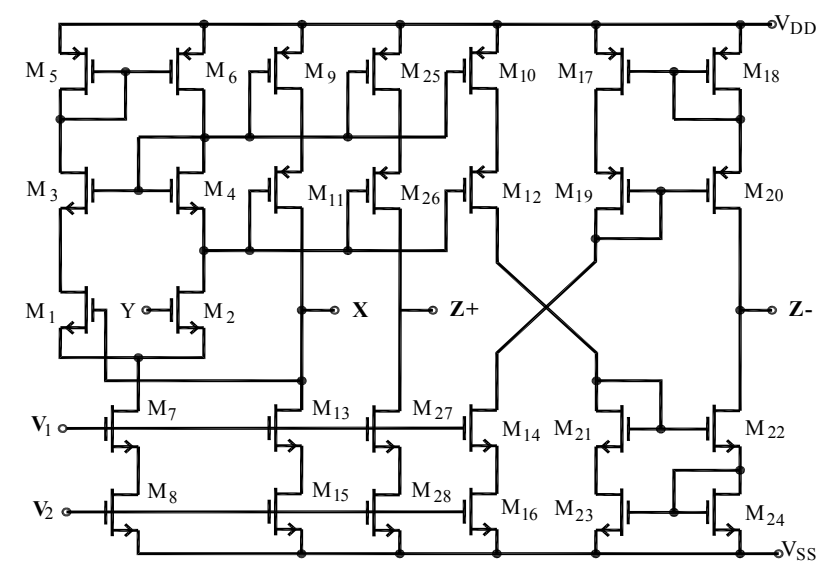

Figure 3. CMOS realization of the CCII.

Table 1. Aspect ratios of CCII MOSFETs.

\begin{tabular}{cc}
\hline MOS transistors & $\mathrm{W} / \mathrm{L}$ \\
\hline$M_{1}-M_{4}$ & $10 / 0.35$ \\
$M_{5}, M_{6}$ & $16 / 0.35$ \\
$M_{7}, M_{8}, M_{13}-M_{16}, M_{21}-M_{24}, M_{27}, M_{28}$ & $16 / 0.35$ \\
$M_{9}-M_{12}, M_{17}-M_{20}, M_{25}, M_{26}$ & $30 / 0.35$ \\
\hline
\end{tabular}

Table 2. Aspect ratios of MO-CCCA MOSFETs.

\begin{tabular}{cc}
\hline MOS transistors & $\mathrm{W} / \mathrm{L}$ \\
\hline$M_{1}-M_{3}$ & $9.5 / 0.55$ \\
$M_{4}-M_{8}, M_{17}, M_{18}, M_{24}-M_{27}$ & $27.5 / 1.5$ \\
$M_{10}-M_{15}, M_{21}-M_{23}$ & $9.5 / 1.35$ \\
$M_{16}, M_{19}, M_{20}$ & $4.5 / 0.7$ \\
\hline
\end{tabular}




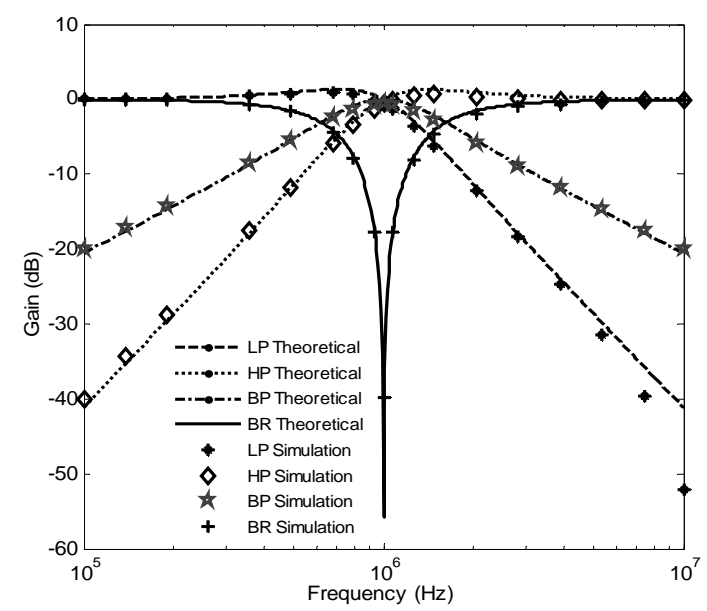

(a)

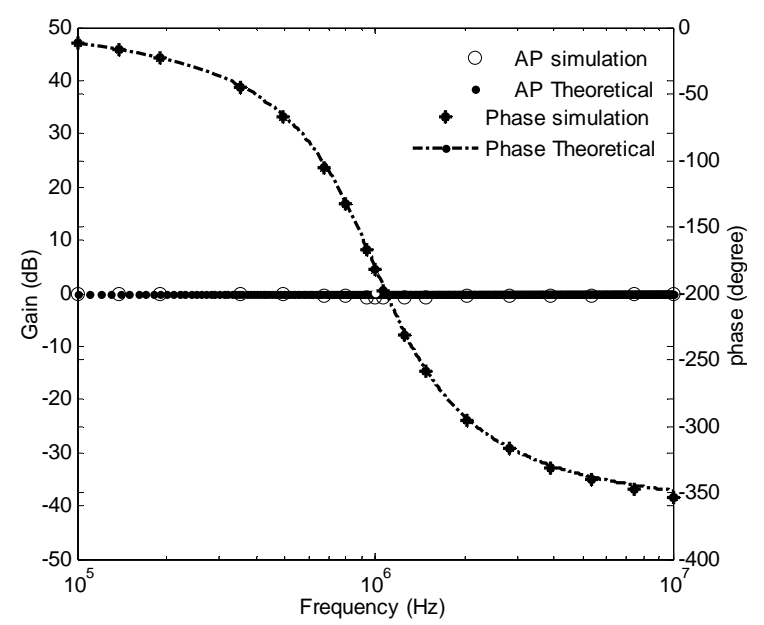

(b)

Figure 4. PSPICE Simulation results (a) Gain response of LPF, BPF, HPF and Notch; (b) Gain and Phase response of APF.

been done for a sinusoidal input signal at $f_{o}=1 \mathrm{MHz}$. Figure 5 shows that the input dynamic range of the filter response extends up to amplitude of $105 \mu \mathrm{A}$ without significant distortion. The dependence of the output harmonic distortion on the input signal amplitude is illustrated in Figure 6. For input signal amplitudes lower than $110 \mu \mathrm{A}$, the total harmonic distortion (THD) is of the order of less than $1 \%$ after that rapidly increasing is occurred. The obtained results show that the circuit operates properly even at signal amplitudes of about $120 \mu \mathrm{A}$ and THD less than $4 \%$.

To achieve the SIMO type filters with $f_{o}=1 \mathrm{MHz}$ and quality factor of $Q_{o}=2$, the component values were selected $K=2\left(n=1, I_{A}=25 \mu \mathrm{A}, I_{B}=100 \mu \mathrm{A}\right)$, $R_{1}=R_{2}=1 \mathrm{k} \Omega$ and $C_{1}=C_{2}=159 \mathrm{pF}$. The circuit realizes LP, HP, and BP responses, respectively, at $i_{01} ; i_{02}$ and $i_{03}$ simultaneously. The frequency responses of Notch and AP can be realized by, respectively, $\left(i_{01}+i_{02}\right)$ and $\left(i_{01}+i_{02}+i_{03}\right)$. Four filter responses are shown in Figure 7.

Figure 8 shows the simulation results for control of $Q_{0}$ while keeping $f_{o}$ fixed $(1 \mathrm{MHz})$ with $C_{1}=C_{2}=159 \mathrm{pF}$ for different values of $Q_{o}$ as shown in Table 3. Figure 9 shows the simulation results for control of $f_{\mathrm{o}}$ while keeping $Q_{0}=1$ with $C_{1}=C_{2}=53 \mathrm{pF}$ for different values of $f_{\mathrm{o}}$ as shown in Table 3 . The current mode band pass filter

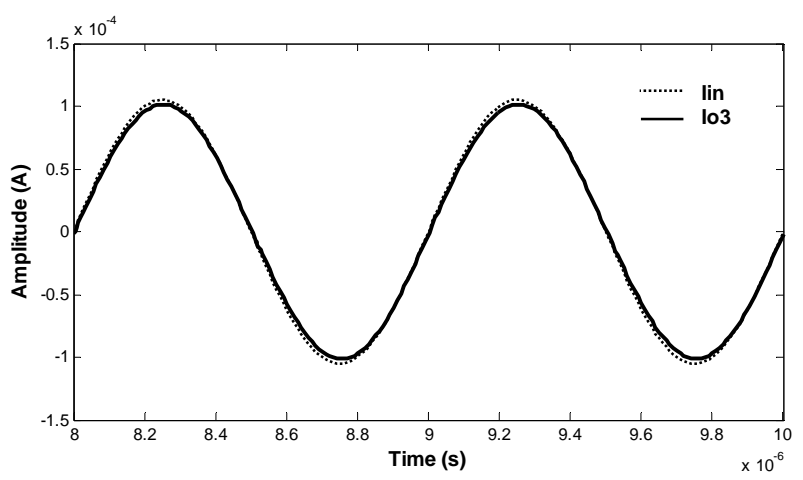

Figure 5. Time domain response of the input and output waveforms of the band-pass filter of the proposed circuit for $1 \mathrm{MHz}$ sinusoidal input current of $105 \mu \mathrm{A}$.

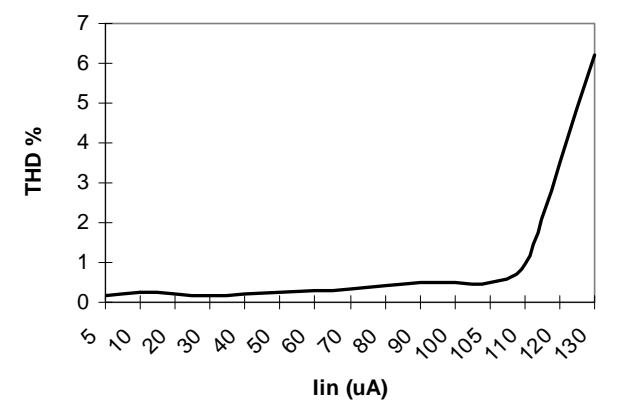

Figure 6. Dependence of output current harmonic distortion on input current amplitude of the band-pass filter of proposed circuit.

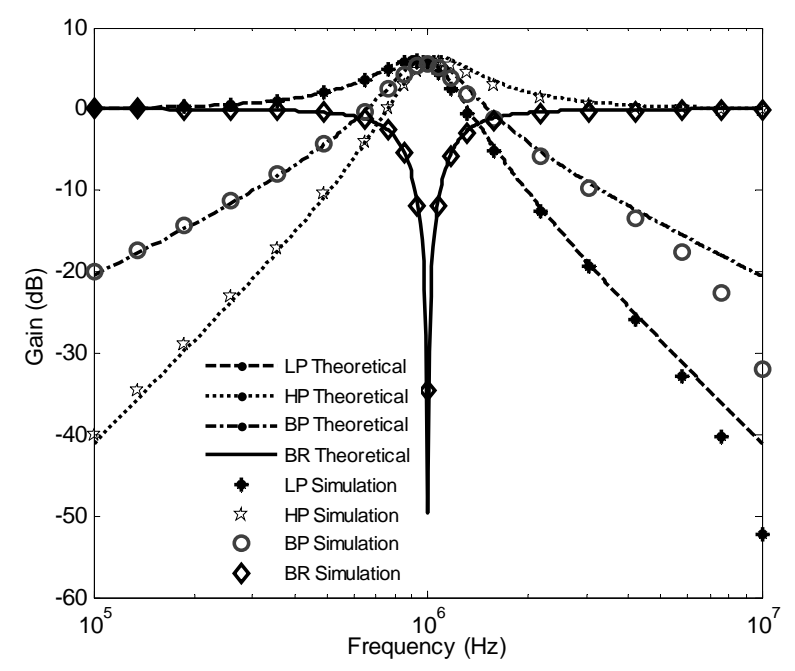

Figure 7. PSPICE Simulated gain responses of LP, BP, HP and Notch for SIMO type filter. 
Table 3. The $R_{1}$ and $R_{2}$ values for controlling of $Q_{o}$ and $C_{1}$ and $C_{2}$ values for controlling $f_{0}$.

\begin{tabular}{cccccc}
\hline & Fixed $f_{o}$ & \multicolumn{4}{c}{ Fixed $Q_{o}$} \\
\hline$Q_{0}$ & $\mathrm{R}_{1} \mathrm{k} \Omega$ & $\mathrm{R}_{2} \mathrm{k} \Omega$ & $f_{0} \mathrm{MHz}$ & $\mathrm{R}_{1} \mathrm{k} \Omega$ & $\mathrm{R}_{2} \mathrm{k} \Omega$ \\
1 & 1 & 1 & 1 & 3 & 3 \\
2 & 0.7 & 1.43 & 2 & 1.5 & 1.5 \\
4 & 0.5 & 2 & 3 & 1 & 1 \\
\hline
\end{tabular}

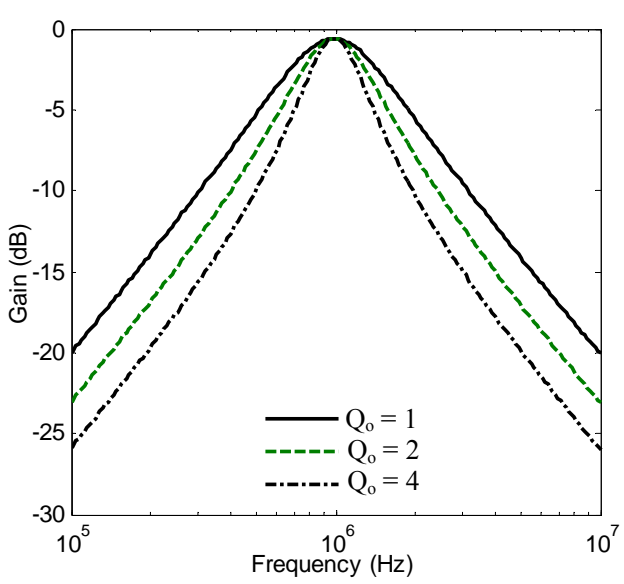

Figure 8. Simulation results for control of $Q_{o}$ while keeping $f_{o}$ fixed (1 MHz) for band pass filter.

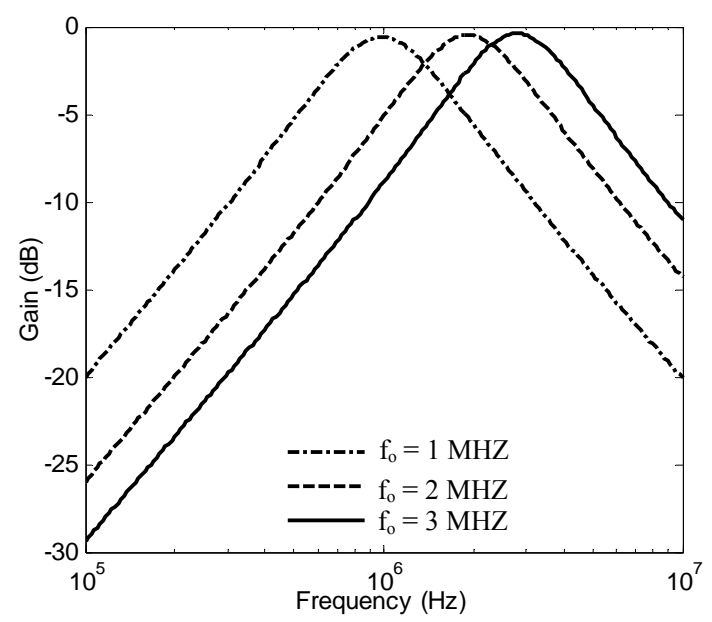

Figure 9. Simulation results for control of $f_{0}$ while keeping $Q_{o}(=1)$ fixed for band pass filter.

is tested for gain and quality factor tuning while keeping pole frequency constant at $1 \mathrm{MHz} . \quad R_{1}=R_{2}=1 \mathrm{k} \Omega$, $C_{1}=C_{2}=159 \mathrm{pF}$ and $K=1,2,4$ are taken for gain = quality factor $=1,2,4$, respectively. The simulated results are shown in Figure 10.

A very good correspondence between design values and values determined from PSPICE simulations is observed, which confirms the workability of the current mode filters realized from the proposed configuration.

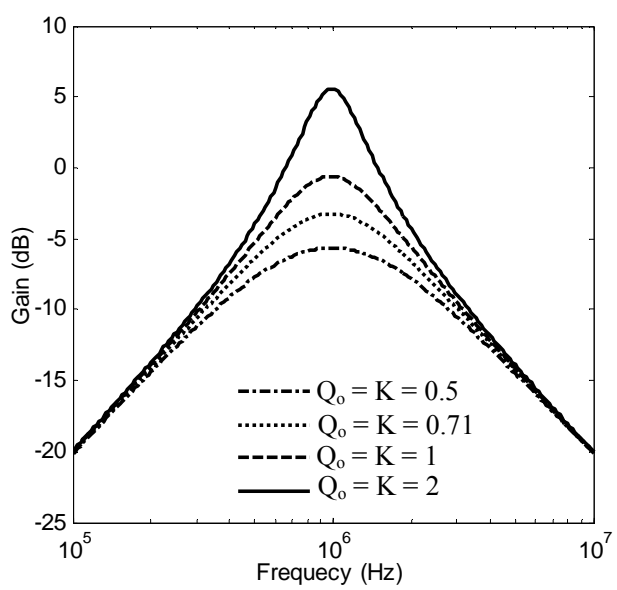

Figure 10. Simulation results for control of $Q_{o}$ and gain while keeping $f_{o}=1 \mathrm{MHz}$ fixed for band pass filter.

\section{Concluding Remarks}

A new universal MISO/SIMO type current-mode biquad employing two DOCCII, one MOCCCA and four passive grounded elements is proposed in this paper. The purpose of this paper as to introduce a new configuration which although uses exactly same number of active and passive components but in contrast to the circuit of reference [1] realizes a MIMO-type biquad and hence, does not require any additional hardware duplicate/invert current inputs which is required in case of MISO-type filters of [1]. The centre frequency $\omega_{0}$ can be set by the passive elements of the circuit and the quality factor $Q_{o}$ is electronically tunable through bias currents of the MOCCCA. Therefore, the biquad filter has independent tenability for the $\omega_{o}$ and $Q_{o}$. The active and passive sensitivities $Q_{o}$ and $\omega_{o}$ are low.

The workability of the new configuration has been demonstrated by PSPICE simulation results based upon a CMOS CCII in $0.35 \mu \mathrm{m}$ technology

\section{REFERENCES}

[1] W. Chunbua, L. Haiguang and Z. Yan, "Universal Current-Mode Filter with Multiple Inputs and One Output Using MOCCII and MO-CCCA," International Journal of Electronics and Communications (AEU), Vol. 63, No. 6, 2009, pp. 448-453. doi:10.1016/j.aeue.2008.03.004

[2] R. Senani and A. K. Singh, "A New Universal Current-Mode Biquad Filter," Frequenz: Journal of TeleCommunications, Vol. 56, No. 1-2, 2002, pp. 55-59.

[3] R. Senani, V. K. Singh, A. K. Singh and D. R. Bhaskar, "Tunable Current-Mode Universal Biquads Employing Only Three MOCCs and All Grounded Passive Elements: Additional New Realizations," Frequenz: Journal of TeleCommunications, Vol. 59, No. 7-8, 2005, pp. 220-224.

[4] R. Senani, A. K. Singh, V. K. Singh, "New Tunable SIMO-Type Current-Mode Universal Biquad Using Only Three MOCCs and All Grounded Passive Elements," 
Frequenz: Journal of Tele-Communications, Vol. 57, No. 7-8, 2003, pp. 160-161.

[5] H. Y. Wang and C. T. Lee, "Versatile Insensitive Current Mode Universal Biquad Implementation Using Current Conveyors," IEEE Transactions on CAS-II. Analog and Digital Signal Processing, Vol. 48, No. 4, 2001, pp. 409413.

[6] W. Chunhua, Z. Yan, Z. Qiujing and D. U. Sichun, "A New Current Mode SIMO-Type Universal Biquad Employing Multi-Output Current Conveyors (MOCCIIs)," Radioengineering, Vol. 18, No.1, 2009, pp. 83-88.

[7] J. W. Horng, C. L. Hou, C. M. Chang, J. Y. Shie and C. H. Chang, "Universal Current Filter with Single Input and Three Outputs Using MOCCIIs," International Journal of Electronics, Vol. 94, No. 4, 2004, pp. 191-197.

[8] M. Siripruchyanun and W. Jaikla, "Cascadable CurrentMode Biquad Filter and Quadrature Oscillator Using DO-CCCIIs and OTA," Circuits Systems and Signal Processing, Vol. 28, No. 1, 2009, pp. 99-110. doi:10.1007/s00034-008-9072-5

[9] R. Senani, "New Universal Current Mode Biquad Employing All Grounded Passive Components but Only Two DOCCs," Journal of Active and Passive Electronic Devices, Vol. 1, No. 3-4, 2006, pp. 281-288.

[10] M. Kumngern, W. Jongchanachavawat and K. Dejhan, "New Electronically Tunable Current-Mode Universal Biquad Filter Using Translinear Current Conveyors," International Journal of Electronics, Vol. 97, No. 5, 2010, pp. 511-523. doi:10.1080/00207210903486823

[11] T. Tsukutani, Y. Sumi and N. Yabuki, "Versatile Current-Mode Biquadratic Circuit Using Only Plus Type
DO-DVCCs and Grounded Passive Capacitors," International Journal of Electronics, Vol. 94, No. 12, 2007, pp. 1147-1156. doi:10.1080/00207210701789998

[12] T. Tsukutani, Y. Sumi and N. Yabuki, "Novel CurrentMode Biquadratic Circuit Using Only Plus Type DODVCCs and Grounded Passive Components," International Journal of Electronics, Vol. 94, No. 12, 2007, pp. 1137-1146. doi:10.1080/00207210701791085

[13] H. P. Chen and P. L. Chu, "Versatile Universal Electronically Tunable Current-Mode Filter Using CCCIIs," IEICE Electronics Express, Vol. 6, No. 2, 2009, pp. 122-128. doi:10.1587/elex.6.122

[14] C. H. Wang, J. Xu, A. Ü. Keskin, S. C. Du and Q. J. Zhang, "A New Current-Mode Current-Controlled SIMOType Universal Filter," International Journal of Electronics and Communications (AEU), Vol. 65, No. 3, 2011, pp. 231-234. doi:10.1016/j.aeue .2010.02. 010

[15] V. K. Singh, A. K. Singh and R. Senani, "Dual Function Capability of Recently Proposed Four-Current Conveyor-Based VM Biquad," Journal of Circuits, Systems and Computers, Vol. 14, No. 1, 2005, pp. 51-56. doi:10.1142/S0218126605002143

[16] S. I. Liu, H. W. Tsao and J. Wu, "CCII-Based Continuous-Time Filters with Reduced Gain Bandwidth Sensitivity," IEEE Proceedings of Circuits, Devices \& Systems, Vol. 139, No. 2, 1991, pp. 210-216.

[17] E. Yuce, S. Minaei and O. Cicekoglu, "A Novel Grounded Inductor Realization Using a Minimum Number of Active and Passive Components," ETRI Journal, Vol. 27, No. 4, 2005, pp. 427-432. doi:10.4218/etrij.05.0104.0149 